\title{
jULIEs: extracellular probes for recordings and stimulation in the structurally and functionally intact mouse brain
}

\author{
Romeo Racz*1-4 ${ }^{* 1}$ Mihaly Kollo*1-4, Gabriella Racz ${ }^{1}$, Ciprian Bulz ${ }^{1}$, Tobias Ackels ${ }^{1,2}$, Tom Warner ${ }^{1}$, \\ William Wray ${ }^{1-4}$, Nikolai Kiskin ${ }^{1}$, Chi Chen ${ }^{6}$, Zhiwen $\mathrm{Ye}^{5}$, Livia de $\mathrm{Hoz}^{6,7}$, Ede Rancz ${ }^{5}$, Andreas \\ Schaefer ${ }^{1-4}$ \\ ${ }^{1}$ Neurophysiology of Behaviour Laboratory, The Francis Crick Institute, London, UK \\ ${ }^{2}$ Department of Neuroscience, Physiology \& Pharmacology, University College London, UK \\ ${ }^{3}$ Behavioral Neurophysiology, Max-Planck-Institute for Medical Research, Heidelberg, Germany \\ ${ }^{4}$ Department of Anatomy and Cell Biology, Faculty of Medicine, University of Heidelberg, \\ Germany \\ ${ }^{5}$ Cortical Circuits Laboratory, The Francis Crick Institute, London, UK \\ ${ }^{6}$ Department of Neurogenetics, Max-Planck-Institute für Experimentelle Medizin, Göttingen, \\ Germany \\ ${ }^{7}$ NWFZ, Charité - Universitätsmedizin Berlin, Germany. \\ * equal contribution
}

Corresponding authors:

romeo.racz@crick.ac.uk, mihaly.kollo@crick.ac.uk, andreas.schaefer@crick.ac.uk

\begin{abstract}
High signal-to-noise, scalable and minimally invasive recording and stimulation of the nervous system in intact animals is of fundamental importance to advance the understanding of brain function. Extracellular electrodes are among the most powerful tools capable of interfacing with large neuronal populations ${ }^{1-3}$. Neuronal tissue damage remains a major limiting factor in scaling electrode arrays, and has been found to correlate with electrode diameter across different electrode materials, such as microfabricated Michigan and Utah-style arrays ${ }^{4}$, MEMS and microsystems $^{5}$, soft polymer or tungsten electrodes ${ }^{6}$ and Parylene $C$ probes ${ }^{7}$. Small diameter ultramicroelectrodes (UMEs), while highly desirable, pose significant technical challenges such as reaching sufficient electrolyte-electrode coupling and limiting stray signal loss. To overcome these challenges, we have designed juxtacellular Ultra-Low Impedance Electrodes (jULIEs), a scalable technique for achieving high signal-to-noise electrical recordings as well as stimulation with UMEs. jULIEs are metal-glass composite UMEs thermally drawn to outer diameters (OD) of $<25 \mu \mathrm{m}$, with metal core diameters (ID) of as little as $1 \mu \mathrm{m}$. We introduce a two-step electrochemical modification strategy that reduces UME coupling impedances by two orders of magnitude. Modifications enabled high signal-to-noise neural recordings in vivo through wires with micrometer scale core diameters. Histological and imaging experiments indicated that local vascular damage is minimal. Spikes reached amplitudes over $1 \mathrm{mV}$ in vivo, indicating that recordings are possible in close proximity to intact neurons. Recording sites can be arranged in arbitrary patterns tailored to various neuroanatomical target structures and allowing parallel penetrations. jULIEs thus represent a versatile platform that allows for reliable recording and manipulation of neural activity in any areas of the functionally intact mammalian brain.
\end{abstract}

\section{Main}

jULIE neural probes were assembled (Fig. 1 and Supplementary Figure 1) from composite glass-metal microwires. These were produced through a modified dieless TaylorUlitovsky drawing method ${ }^{8,9}$ (Fig.1a). Fibers were drawn from a gold or copper-filled Pyrex glass 
preform prepared under inert atmosphere and heated to melting temperatures until a glassmetal droplet formed. The glass capillary was pulled and spooled up at high speeds (up to $2 \mathrm{~km} / \mathrm{min}$ ) on a drum. Depending on pulling force and drum speed, core diameters ranged between $<1$ and $10 \mu \mathrm{m}$ with glass insulation thickness up to $15 \mu \mathrm{m}$. The resulting microwires were thus small (Fig.1b), well insulated (Fig.1c,j) and continuously conductive over several hundred meters, yet remained flexible with $\sim 500 \mu \mathrm{m}$ bending radius (Fig.1d). They featured smooth exteriors (Fig.1C) and had well-defined glass-metal boundaries (Fig.1j). Before assembling into recording arrays (Supplementary figure 1) individual wires were prepared as follows. Wires were rewound from the spooling drum. Stacks of wires were then embedded in a soluble thermoplastic (see methods) and polished at sharp angles of approximately 30 degrees (Fig.1c, Supplementary figure $1 \mathrm{a}, \mathrm{b}, \mathrm{c}$ ) to facilitate insertion and penetration into tissue ${ }^{10,11}$.

Given the small area of the conductive core and resulting small electrical interface with the extracellular space, impedance $|\mathrm{Z}|$ of the bare metal core was too high for neural recordings across frequencies from $1 \mathrm{~Hz}$ to $100 \mathrm{kHz}$ (Fig. 1n,o) resulting in signal attenuation and increased noise ${ }^{12-14}$. This is a common problem of ultra-thin electrodes. Electrode-tissue coupling can, in general, be improved through alteration of the surface by physical, chemical and electrochemical means, albeit often resulting in uncontrolled geometrical alterations and mechanical failure ${ }^{15}$.

Both organic and inorganic materials have been used to reduce electrode impedance for larger electrode surfaces ${ }^{16}$. Iridium oxide (IrOx) electrodeposition for example is a wellestablished surface modification for extracellular recording electrodes ${ }^{17-19}$. Thanks to its faradaic nature and stable reversible $\mathrm{Ir}^{3+} / \mathrm{Ir}^{4+}$ redox couple it also provides an excellent substrate for microstimulation ${ }^{20}$. Here, we modified jULIEs with an initial nanostructured gold then further added a nanostructured IrOx layer to further optimize coupling to the extracellular signal.

To maintain the cylindrical profile and small footprint of the jULIE tip, our aim was to increase the specific surface area of the metal sensor and coupling to the extracellular voltage signal while keeping geometrical size increase controlled and minimal. We therefore established a three-step microwire surface modification protocol (Fig.1j-p, Supplementary figure 1-3): firstly, as a result of the polishing described above (Supplementary figure 1a-c) the metal core was exposed in a controlled and reproducible manner (Fig.1j). Secondly, after de-embedding, the exposed core was modified with high-rugosity gold nanostructures (nanoAu) (Fig.1k, Supplementary Figure 2) by electrodeposition from an additive free cyanide electrolyte (see methods). While microwires can be produced from a wide range of metals, potential chemical incompatibility with the tissue may occur, causing toxicity or corrosion. The deposition of gold nanostructures decouples the electrode-electrolyte interface from the core metal, allowing the use of custom conductive materials for the core, largely independent of their toxicity or corrosion properties. In the following, third step, gold nanostructures were further modified with a porous film of polycrystalline IrOx (Fig.1I, Supplementary figure 3) from an aged $\mathrm{IrCl}_{4}$ electrolyte by a combined cyclic voltammetry and potentiostatic electrodeposition protocol. During the first sweeps of electrodeposition, in case of transition metal oxides, nucleation centers develop (as shown e.g. for $\mathrm{MnO}_{2}{ }^{21}$ ) and charge is being stored with each voltammetry cycle. Similarly, for IrOx (Supplementary figure 3a) cyclic voltammetry facilitated development of IrOx nucleation centers on nanoAu; subsequent potentiostatic pulses further accelerated buildup (Supplementary figure $3 \mathrm{~b}$ ).

The resulting films of polycrystalline IrOx (Supplementary figure 3d-f) evenly covered the nanoAu substrate (Fig. 1l). Depending on the dimensions of the nanoAu deposit (Fig. $1 \mathrm{~m}$ ) and IrOx film thickness, in combination, nanoAu+IrOx reduced interfacial impedances up to 100 times (Fig. $1 \mathrm{n}, \mathrm{o})$ to values of around $100 \mathrm{k} \Omega$ at $1 \mathrm{kHz}$. These values were adjustable through modulation 
of electrodeposition parameters (Fig. $1 \mathrm{~m}$ ) to match e.g. size constraints or input impedances of amplifiers.
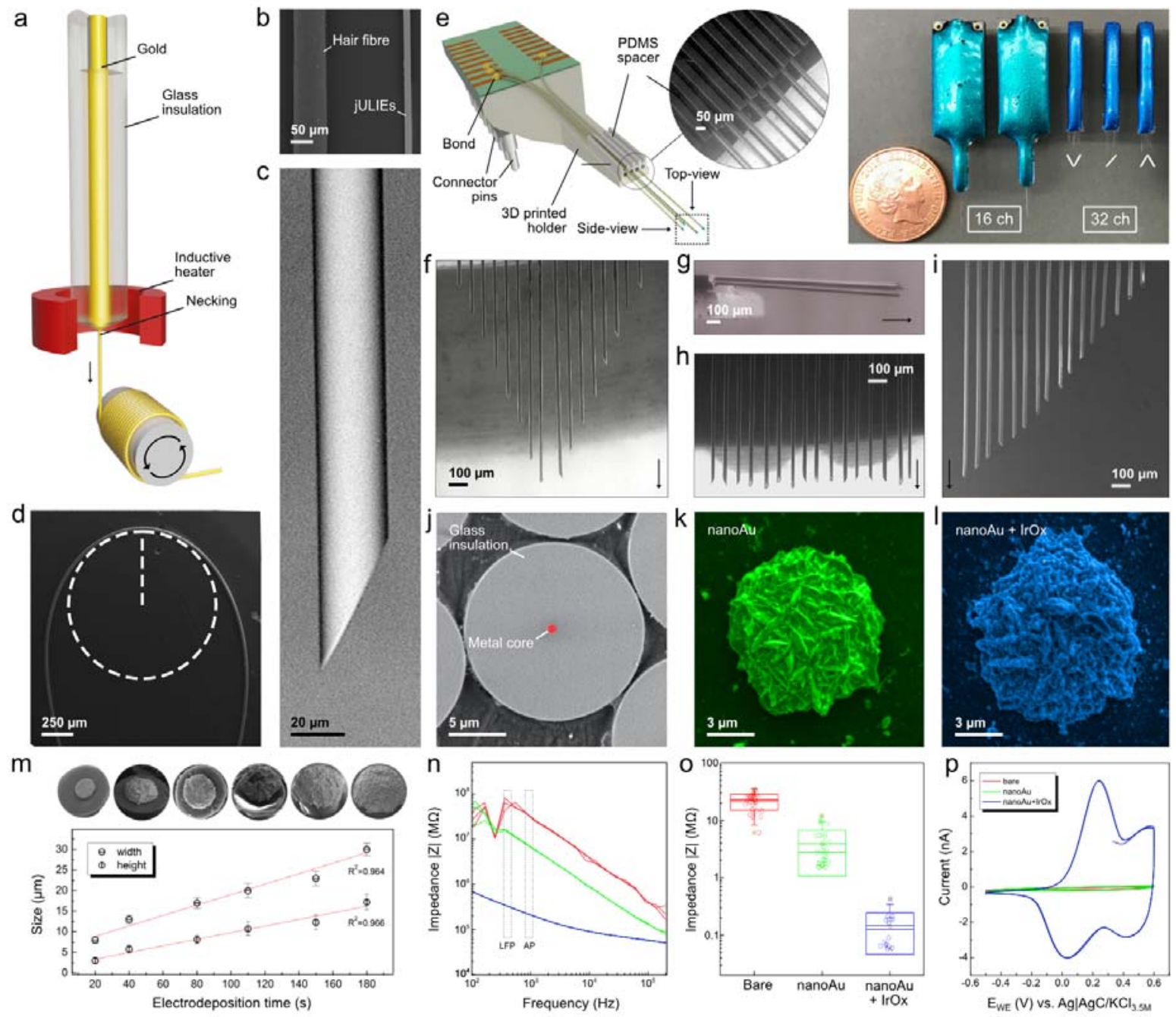

Figure 1. (a) Apparatus and principle of glass-insulated ultramicroelectrode fabrication by high-speed dieless drawing. A preform containing the metal core and glass insulation is heated and the drop formed at the necking is continuously spooled up on a drum. (b) SEM image comparing diameters of a human hair and a jULIE fiber. (c) Side-view SEM of a sharpened wire before electrochemical preparation. (d) SEM image of the bending radius of approx. $500 \mu \mathrm{m}$ of a single fiber. (e) 3D CAD model and actual assembled jULIEs with custom layout of 16 and 32 channels and standard 128 channel probes with gold and copper cores. (f-i) Top-view SEM micrographs of customized recording site layouts featuring different planar tip geometries with laterally dense packing at $50 \mu \mathrm{m}$ inter-shank distance. (j-l) Front-view SEM of flat polished bare fiber $(\mathbf{j})$, modified with nanoAu for $35 \mathrm{~s}(\mathbf{k})$ and nanoAu modified with IrOx (l). (m) SEM micrograph of progressive alteration of the initial core with nanoAu by controlling electrodeposition time, nanoAu building up preferentially as a hemisphere. Electrochemical impedance spectroscopy of surface preparation stages $(j-1)$ : $(n)$ Impedance $Z$ Bode-plot between $1 \mathrm{~Hz}$ and $100 \mathrm{kHz}$, (o) impedance at $1 \mathrm{kHz}$ and (p) cyclic voltammetry response at 50 $\mathrm{mVs}^{-1}$ of the three preparation stages.

Surface modifications were mechanically stable during manipulation, tissue insertion, and even penetration in deep brain structures $(\sim 1300 \mu \mathrm{m}$, Supplementary figure 4$)$. 
For extracellular recordings, microwires were assembled into low-profile custom 3D printed polymer holders incorporating a custom printed circuit board (PCB) and connector plug with matched channel count and a spacer separating individual fibers (Fig.1e and Supplementary figure 1). To record from distributed ensembles of neurons, we connected groups of 10-128 wires to custom designed PCBs using a modified ball-bonding technique (Supplementary figure 7). In this semi-automatic process, individual wires were placed in arbitrary 2D and 3D arrangements, at different depths with lateral spacing varying between 5 to $150 \mu \mathrm{m}$ (shown for $50 \mu \mathrm{m}$ inter-shank separation in Fig.1e-i). The overall layout of recording sites was tailored to the neuroanatomical structure of interest.

\section{Results}

\section{Minimally invasive electrodes}

To validate the technology for extracellular recording and stimulation, we inserted assembled jULIEs into the olfactory bulb (OB) of 4-6-week-old anaesthetized mice (Fig. 2a-g). They reliably resolved single and multi-unit activity immediately after insertion starting from superficial layers (Fig. 2e). This was in contrast to standard microfabricated probe recordings where unit activity is typically suppressed at up to $\sim 45$ minutes after probe insertion ${ }^{22}$ which suggests that tissue integrity is better maintained upon jULIEs insertion.

To directly assess the integrity of the vasculature, we coated jULIEs with DiO, labeled the blood vessels with sulforhodamine, and monitored tissue structure during insertion using 2photon microscopy. While individual fibers were stiff enough to penetrate, they were sufficiently compliant to preserve both the structure of the tissue, with capillaries folding around the fiber shank, and slide on its surface without dragging the surrounding tissue, or apparent rupture of the vessels while inserted (Fig.2a and Supplementary video 1). To assess the latter more directly we performed histology after insertion experiments where the vasculature was loaded with Evans-Blue (Fig. 2 b, c), a dye unable to penetrate the blood-brain barrier due to its high-affinity binding to the large protein albumin ${ }^{23}$. For standard silicon probes (Fig. 2b) insertion resulted in significant local damage to the blood-brain-barrier as measured by extravasation of Evans Blue (Fig $\mathbf{2} \mathbf{b}_{2}$ ) and tissue displacement with loss of neuron density around the rectangular shank (Fig $2 \mathrm{~b}_{3}$ ). Insertion of the jULIEs, on the other hand (Fig.2c), left cell density seemingly unperturbed (Fig. $2 \mathrm{c}_{3}$ ) and resulted in no detectable damage to the bloodbrain barrier (Fig. $2 c_{2}$ ), suggesting that the neural tissue had been left structurally intact.

\section{In-vivo extracellular recordings}

We recorded spontaneous single and multi-unit activity with high signal-to-noise ratio while slowly inserting jULIEs into the main olfactory bulb (MOB) (Fig.2, Supplementary figure 5). At insertion speeds of $\sim 5 \mu \mathrm{m} / \mathrm{s}$ we readily detected the emergence of unit activity (Fig.2d,e). Action potential (AP) amplitude increased from background levels to its maximum as jULIEs were advanced by 10-100 $\mu \mathrm{m}$ and subsequently decreased over similar length scales with further insertion. Highest amplitudes exceeded $1 \mathrm{mV}$ suggesting close proximity of the recording site to the intact neuron at that point ${ }^{24,25}$.

When jULIEs were displaced from the surface towards the center of the olfactory bulb, we found that spiking activity emerged and disappeared in a symmetrical fashion, consistent with the recording electrodes passing through the volume without mechanically dragging the tissue, while capillaries folded and slid around the smooth shank (Supplementary video 1). When jULIEs were inserted and retracted multiple times on the same axis around a spontaneously firing unit, activity was preserved at the same location over several insertionretraction cycles extending beyond the recorded units by several hundred microns, suggesting 
that the local neural network remained functionally intact, and the electrode neither caused significant damage to nor displaced neurons (Fig.2f). Using a jULIE array formed by two stacked layers of nine probes each with $50 \mu \mathrm{m}$ inter-shank spacing and $100 \mu \mathrm{m}$ inter-layer distance, single active units were resolved on several adjacent channels (Fig. 2 g). It is to be noted that such arrangements enabled the usage of well-established spike-sorting algorithms such as Klustakwik $^{26}$, KiloSort ${ }^{27}$ or Plexon Offline Sorter (Plexon Inc.).
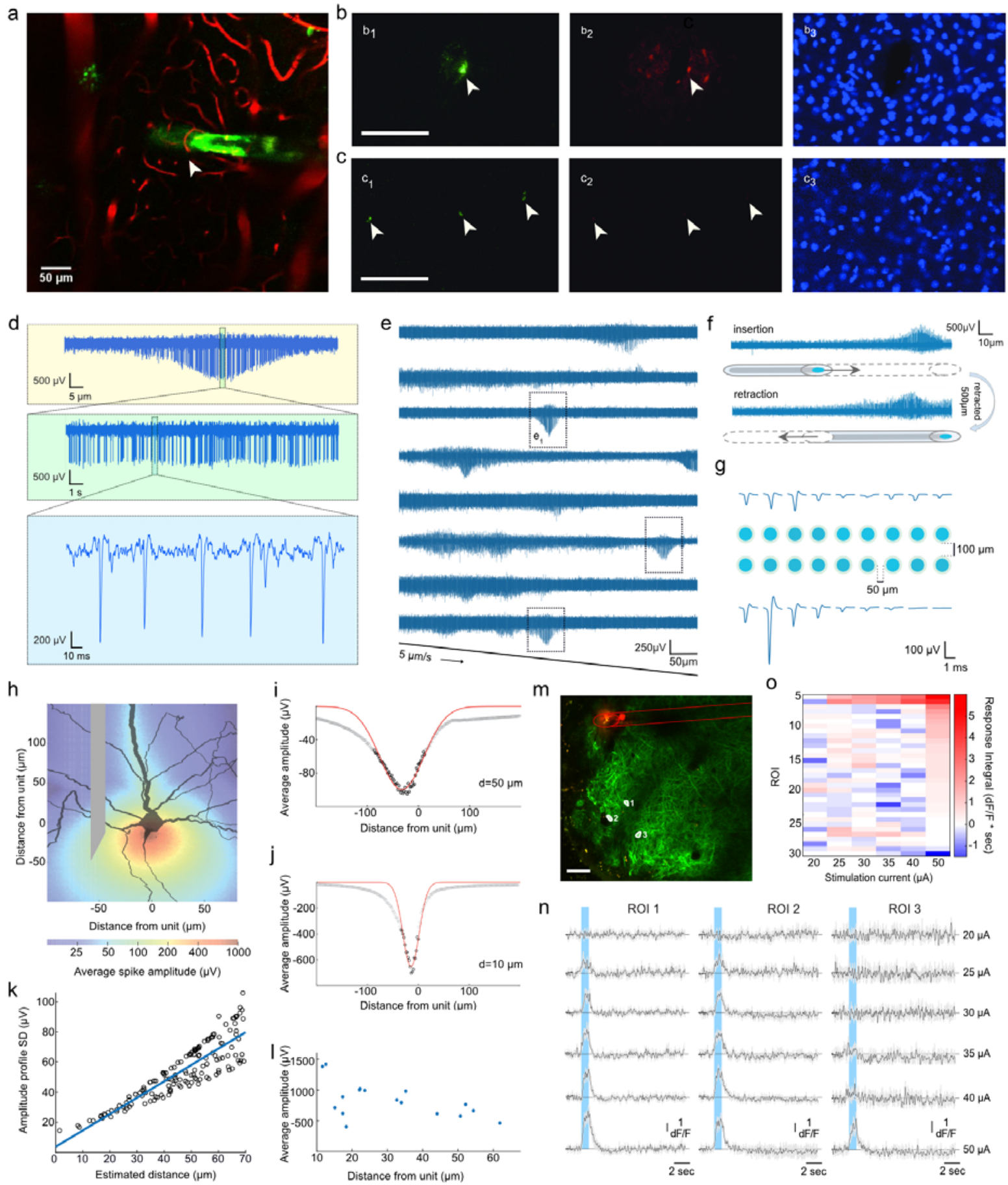

0
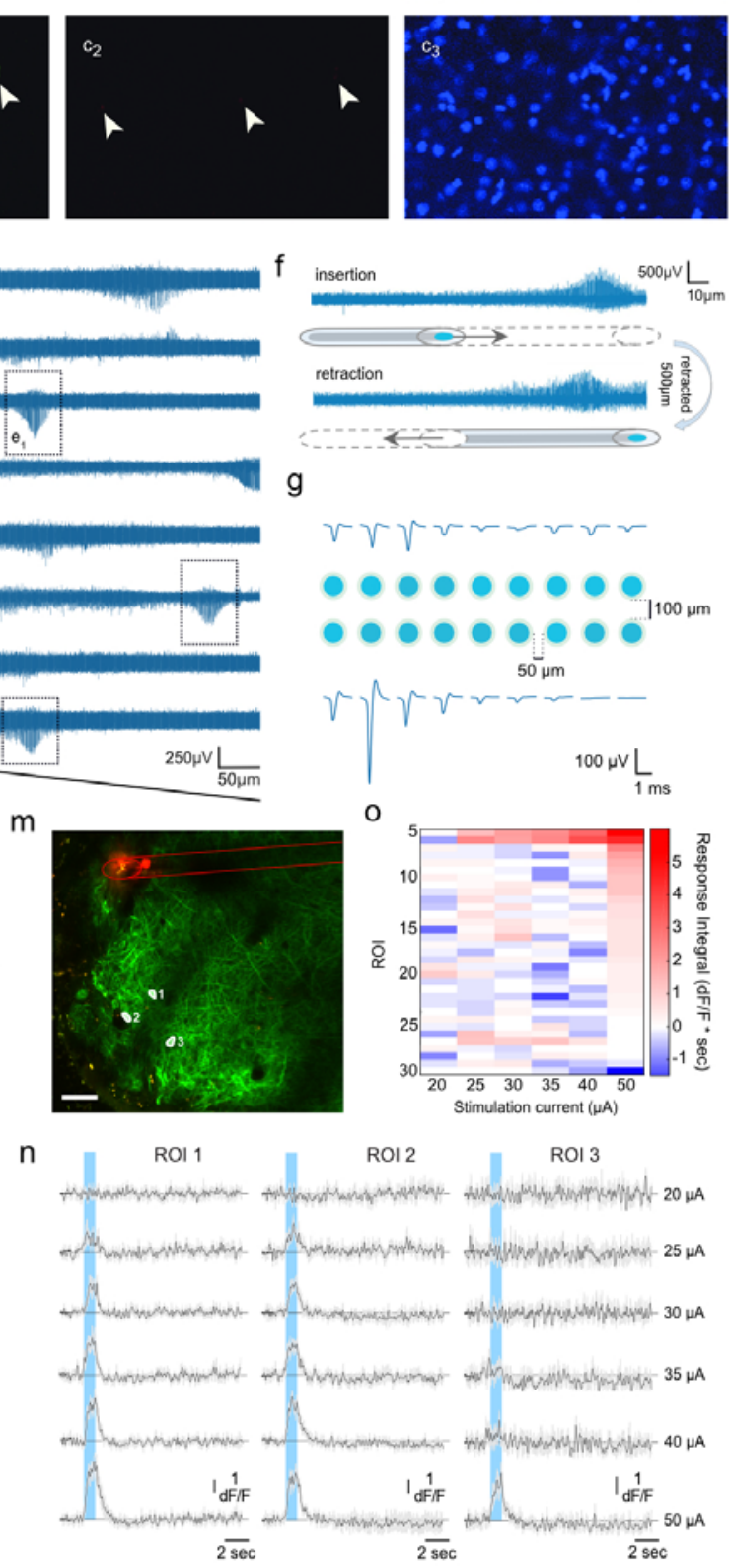

Figure 2. (a) In-vivo acute 2-photon microscopy observation of insertion of a DiO-labeled jULIE (green) into the MOB with sulforhodamine labeled capillaries (red). The jULIE was moved along its axis towards the middle of the OB then retracted several times (see Supplementary Video 
1). (b) Acute tissue damage upon insertion of a Si-probe $\left(b_{1}-b_{3}\right)$ and of a multi-shank jULIE probe $\left(c_{1}-c_{3}\right.$; three jULIEs shown). Insertion sites are labelled by DiO ( $b_{1}, c_{1}$; green). Damage to the blood brain barrier upon Si-probe insertion is indicated by the extravasation of albuminbound EvansBlue ( $b_{2}, c_{2}$; red), and displacement of cell nuclei ( $b_{3}, c_{3}$; DAPI, blue). (d) Details of extracellular recording on a single channel during electrode insertion at constant speed $\sim 5$ $\mu \mathrm{m} / \mathrm{s}$ near a spontaneously firing unit. (e) Spontaneous activity on larger scale in the MOB recorded as multi and single-unit activity $\left(e_{1}\right)$ while jULIEs were inserted $\sim 400 \mu \mathrm{m}$ into the OB. Spontaneously active units appeared as humps of increased amplitude on top of the multi-unit activity. (f) Spontaneous activity was preserved when jULIEs were inserted and retracted multiple times along the same axis in the vicinity of a spontaneously active unit; unit activity was recovered in comparable location during insertion and retraction (despite $\sim 500 \mu \mathrm{m}$ further advancement of the jULIE). (g) Single-unit amplitude distribution on a custom arranged rectangular recording site layout featuring constant $50 \mu \mathrm{m}$ lateral inter-shank and $100 \mu \mathrm{m}$ interlayer distance. (h) Heatmap of simulated spike peak amplitudes at different positions around a mitral cell. (i,j) Modelled spike amplitudes (black circles) at different positions along the electrode axis, and their Gaussian fit (red line) at $50 \mu \mathrm{m}$ (i) and $10 \mu \mathrm{m}$ (j) from the initial segment of biophysically realistic mitral cell. (k) Relationship of the standard deviation of fitted spike amplitudes profiles and the distance of the electrode axis to the initial segment of the neuron. (I) Peak amplitudes of maximal recorded action potentials from 17 jULIE probes and their estimated distance from the mitral cell initial segment, collected experimentally in the MOB. (m-o) Electrical stimulation in vivo in the OB using a single jULIE probe $(\mathrm{m})$ In-vivo DiO labeled position of the jULIE stimulation site relative to ROls labeled $1,2,3$; $(\mathrm{n})$ responses of the 3 ROls indicated in $(\mathrm{m})$ to increasing stimulation current; $(0)$ and response of all identified cells contained within the field of view $(m)$ to increasing stimulus current.

\section{Electrode - neuron distance estimation}

To more precisely estimate the position of jULIEs in relation to the recorded neurons, we calculated the electrical field around biophysically realistic models of mitral cells (Fig. $2 \mathrm{~h}$ ), and estimated the resulting spike amplitudes during axial movement of the electrode (Fig. $2 \mathbf{i}, \mathbf{j}$ ). Simulations of the extracellular electrical field demonstrated that the distance between the axis of electrode movement and the soma of the neuron could be predicted from the standard deviation of the amplitude profile (Fig. 2i-k). We thus performed further electrode insertions into the olfactory bulb while monitoring the change of spike amplitude during motion, and estimated the distance from the recorded units (Fig.2l). We found that reliable recordings could be made at estimated (horizontal) distances as low as $10 \mu \mathrm{m}$ from the axon initial segment. This suggests that sharpened, electrochemically modified microwires could indeed record in close proximity to intact neurons (hence juxtacellular Ultra-Low-Impedance Electrodes, jULIEs).

\section{Stimulation}

A key advantage of the multi-step electrochemical modification described here is the large charge storage capacity due to the increased surface area and the stable reversibility of the $\mathrm{Ir}^{3+} / \mathrm{Ir}^{4+}$ redox couple. To assess whether this would indeed allow for electrical stimulation efficient enough to drive neuronal activity with ultramicroelectrodes, we performed systematic microstimulation under 2-photon observation in the olfactory bulb of transgenic mice expressing the genetically encoded $\mathrm{Ca}^{2+}$ indicator GCaMP6f in projection neurons (Tbet-Cre $x$ Ai95). Using a single jULIE fiber (Fig. $2 \mathrm{~m}$ ) 5-10x 1 ms pulses were injected carrying currents from $2 \mu \mathrm{A}$ to $150 \mu \mathrm{A}$ in $5 \mu \mathrm{A}$ increments (see methods). Cells were found to respond to increasing stimulation levels with most cells in the proximity of the stimulation site responding at $50 \mu \mathrm{A}$ injection current (Fig. $2 \mathrm{~m}-\mathrm{o}$ ). Electrical properties of the interface remained stable throughout 200,000 injected 
voltage pulses (Supplementary figure 6a). Moreover, electrochemical impedance spectroscopy and cyclic voltammetry characterization after current stimulation revealed only a threefold increase after 1 million pulses (Supplementary figures $6 c, d$ ), enabling the use of the same site for further recordings.

\section{Custom site layout}

Silicon-probes are widely adopted because of their ability to perform extracellular recordings with relative ease on multiple channels. However, the underlying microfabrication methods have limitations in terms of materials, dimensions and required efforts to customize sensor layout to fit a certain target brain structure. In contrast, the layout of the recording sites of jULIEs can be readily customized (Fig. $1 \mathrm{e}-\mathrm{i}$ ) to best match anatomical requirements of individual experiments. Microwires can be pre-arranged to maximize site lateral density using micron-scale polymer templates (Supplementary figure 1) which help splay fibers at insertion (Supplementary video 2). To record from sensory areas in the mouse brain, 16-channel prearranged probes were assembled: recording site layout was mapped onto the tonotopical arrangement in the auditory inferior colliculus (IC); or, as an evenly spaced one-layer arrangement, onto L5 neurons in the primary visual cortex.

\section{Recordings in the inferior colliculus}

When sampling auditory physiology, it is often desirable to record along the tonotopic axis to resolve population activity evoked by different frequency components of an auditory stimulus. This often requires multi-shank probes which can be challenging in mice, as tissue damage around the multiple insertion sites is thought to limit the number of successfully recorded units ${ }^{5,28,29}$. Thus, to sample from the 3-dimensional volume of the IC, a 4-layer stacked jULIE probe with a rectangular footprint (Fig.3a) was assembled. Individual probe shanks (Fig.3b) were separated horizontally by $\sim 100 \mu \mathrm{m}$, recording sites were vertically arranged at 100 $\mu \mathrm{m}$ steps matching the slope of the isofrequency planes and inserted into the IC of anaesthetized mice with the deepest shank next to the medial axis, (see Fig.3c). Unit activity in the IC was readily resolved (Fig. 3b3) with presentation of pure tones. Notably, while recordings from superficial layers are generally difficult to obtain ${ }^{30}$, jULIEs allowed to record evoked spiking activity with clear tuning in superficial layers of the IC already at $\sim 40 \mu \mathrm{m}$ from the surface (Fig.3d). Overall, the probe mapped onto the tonotopy of the IC (Fig.3e,f) allows to record tone evoked responses in units across the 2-dimensional surface at different depths (Fig.3,f and $f^{*}$ ), including at very superficial layers.

\section{Recordings in the visual cortex}

The neocortex is a layered structure where targeting individual layers with many recording sites for extracellular recordings is challenging, yet often desirable. While e.g. Sipolytrodes $^{31}$ can achieve single layer multi-channel recordings in cortex, their larger footprint results in local tissue damage and limits their versatility. jULIEs in turn can be arranged in arbitrary 2D and 3D patterns, such as a single-layer horizontal site layout (Fig. $3 g$ ), to target recording sites to individual layers. Recording sites can be spaced as close as necessary, not limited by the number of shanks.

We tested the utility of the laterally dense probes by recording from the primary visual cortex (V1) of lightly anaesthetized mice at cortical depths corresponding to layer 5 (500-750 $\mu \mathrm{m}$ ). Following surgery to expose the cortex, a jULIE probe (16 wires arranged horizontally with $50 \mu \mathrm{m}$ inter-shank spacing) was lowered into the cortex. Separate recordings were performed under similar conditions with a comparable 16-channel Si-probe (4 shanks $150 \mu \mathrm{m}$ apart, each 
containing 4 recording sites in a tetrode arrangement, see Online Methods). Several recording sessions, at least $50 \mu \mathrm{m}$ apart in depth, were made both with the jULIEs and the Si-probe.

Recordings from individual jULIEs had lower noise (RMS $3.77 \pm 0.69 \mu \mathrm{V} ; n=15$ wires) compared to the Si-probe used in this experiment (RMS $12.88 \pm 2.64 \mu \mathrm{V} ; \mathrm{n}=15$ sites, Fig. $3 \mathrm{~h}$ ). Single units appeared on multiple wires (e.g. unit7, Fig. 3i), and the same wire could record from multiple single units (e.g. ch15, Fig.3i). Average spike amplitudes of well-separated units showed a comparable distribution to those from the Si-probe (Fig. $3 \mathrm{~h}, \mathrm{jULIEs:} 66.39 \pm 45.48 \mu \mathrm{V}, \mathrm{n}$ = 33; Si-probe: $77.44 \pm 40.40 \mu \mathrm{V}, \mathrm{n}=39 ; p=0.28$, Student's t-test).

a

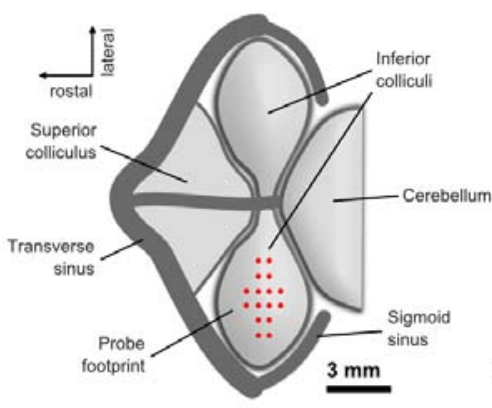

d

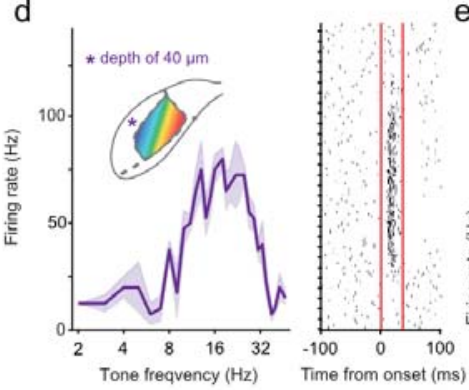

g

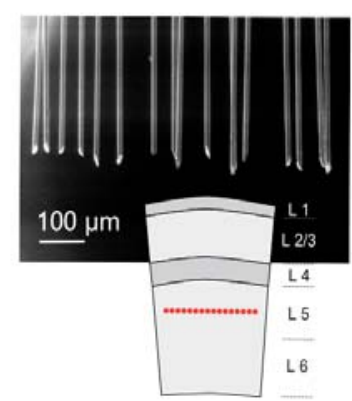

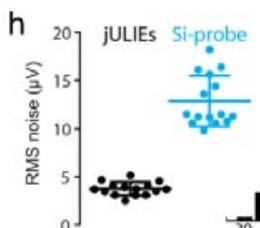
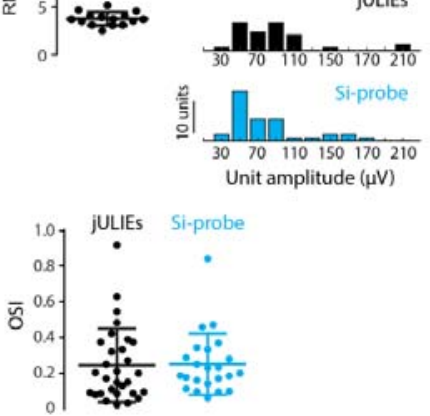

e

i
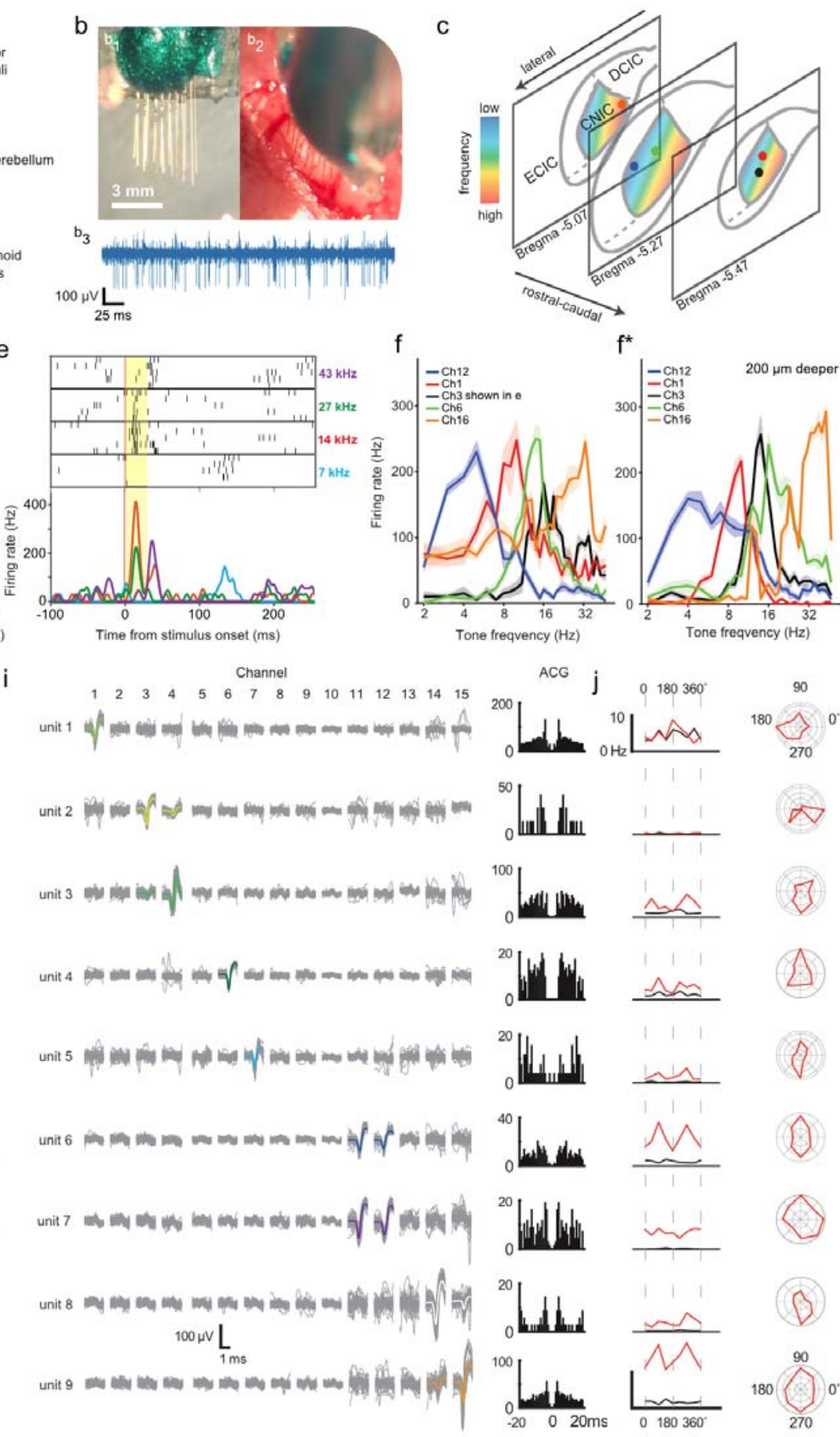

Figure 3. (a-f) jULIE recordings in inferior colliculus (a) Dorsal view of the inferior colliculus 
(IC) as seen after craniotomy. (b1), Side image of the 16-channel jULIE probe; (b2), top view of probe entering the dorsal surface of the IC; (b3), voltage trace with units recorded from IC. (c) spatial distribution of recording sites used for the analysis in $f$ and $f^{*}$. Colors illustrate the arrangement of tonotopy in the IC, with low frequencies best represented in superficial / lateral regions and progressively higher frequencies in progressively deeper / more medial regions. The color of the dots in (c) corresponds to the color of the tuning curves shown in ( $f$ ) and $\left(f^{*}\right)$. (d) jULIE recording from a superficial location $(\sim 40 \mu \mathrm{m}$ below dorsal surface; inset: schematic of recording location in IC dorsal cortex). Note the unimodal frequency tuning (left) and reliable, low-latency response (right); each dot represents a spike, red lines represent stimulus onset and offset respectively). (e) Evoked responses recorded from the same channel in the same location in response to 4 different tones (frequencies colored shown at the side of the raster plot; red line: stimulus onset; stimulus duration: yellow box), each repeated 5 times. Different frequencies evoked different response patterns. While 14 and $27 \mathrm{kHz}$ elicited short-latency responses, $7 \mathrm{kHz}$ elicited no response and $43 \mathrm{kHz}$ elicited a late response. Sparse spontaneous activity is seen before and after the sound presentation. (f) Tuning curves (average of activity evoked by 5 sound presentations) recorded at different locations (colorcoded in c) illustrating position-specific preferred frequencies. Tuning curves are pronounced, unimodal, and discriminable between units, despite being recorded simultaneously from several different positions in the inferior colliculus. $\left(\mathrm{f}^{*}\right)$ Moving the jULIEs $200 \mu \mathrm{m}$ deeper maintained receptive field properties. (g-j) jULIE recordings in visual cortex. (g) SEM micrograph of the recording site layout and their positioning in layer 5 (red dots in the bottom schematic). (h) jULIEs vs. Si-probe: RMS noise distribution, histogram of average spike amplitudes (negative deflection) from single units and distribution of orientation selectivity index (calculated from j) for all units recorded using jULIEs and Si-probe. (i) left: Waveforms of all units separated during a single jULIE penetration (650 $\mu \mathrm{m}$ depth). Average spike waveforms shown in color. Right: Auto-correlograms (ACG) of spike times for separated units. (j) left: Visual responses for the units shown in (i) for 8 different drifting directions (red) and baseline firing rate (black). Asterisks denote significant evoked activity in at least one direction. Right: Polar plots showing normalized tuning curves.

Well-isolated single units (see Online Methods for sorting) could be detected on single or multiple (usually 2 ) neighboring recording sites (Fig. 3i). We recorded visually evoked single-unit activity using standard drifting gratings (8 directions, see Online Methods for details). As expected $^{32}$, activity evoked by visual stimuli showed characteristic tuning curves (Fig. $\mathbf{3 j}$ right) for single units. Units recorded on the same channel (e.g. units 6 and 7) had different levels of evoked activity and markedly different tuning, consistent with the assumption that units represent individual neurons. Furthermore, drifting-grating responsive single units recorded from jULIEs and the Si-probe had similar distribution of orientation selectivity index (jULIEs OSI: 0.24 $\pm 0.20, n=30$ cells; Si-probe OSI: $0.25 \pm 0.17, n=23$ cells; $p=0.88$, Student's t-test, Fig. $3 \mathrm{~h}$ ).

\section{Discussion}

Here we have described the manufacturing, modification and use of "jULIEs", glass-metal composite ultramicroelectrodes, as a minimally invasive and scalable approach to record highquality extracellular signals from the intact mouse brain. We demonstrated that electrochemical multi-step modifications with nanomaterials substantially reduced interfacial impedances, decoupling wire dimensions and materials from electrode-electrolyte coupling. jULIEs were compliant with the tissue structure, causing no detectable tissue displacement or damage to the 
blood-brain-barrier, making them an ideal candidate for long-term implantation. This was likely due to the overall small dimension of individual fibers ${ }^{29,33}$, cylindrical form, sharpened tip and smooth shanks which have been suggested to keep mechanical interaction with surrounding tissue minimal ${ }^{10}$. Other advantages of modified microwires are their composite nature: metal cores are effectively fused with the glass ensheathing providing a robust, defect and delamination-free very low parasitic-capacitance insulation ${ }^{34}$. Polymer coated microwires ${ }^{35}$, microfabricated polymer electrodes ${ }^{36-38}$, carbon fibers ${ }^{33,39}$, multimodal pipettes ${ }^{40}$, syringeinjectable mesh electrodes ${ }^{41,42}$ and niobium microwires ${ }^{43}$, amorphous $\mathrm{SiC}^{44}$ and active-CMOS probes $^{45}$ are promising avenues for potentially low-damage neural recordings. Several approaches have been put forward to allow for their connectorization to standard readout electronics however at very large scale (above 10k) these still remain a challenge. Due to their metal core, the jULIEs can be readily connected to integrated read-out electronics in a scalable manner, either through direct bonding on a smaller scale (Supplementary Fig 7) or through nonthermal flip-chip bonding at very large scale ${ }^{11,34}$. Moreover, when assembled into customized neural probes, the overall layout of sensor sites can be individually tailored to fit the neuroanatomical structure in three dimensions.

Multi-step electrochemistry enables decoupling of the recording interface in contact with the neuronal tissue from the material properties of the wires and their limitations. This might further allow usage of core compositions which improve wetting capabilities needed to manufacture thinner fibers ${ }^{46}$. NanoAu deposition could then restore the contact surface area and electrochemical compatibility, and, in tandem with IrOx deposition, provide a low impedance electrode-electrolyte interface despite submicron core diameters. In fact, microwire modification with an inert metal such as the nanoAu enables access to a diverse set of impedance reducing strategies with materials such as polymers, carbonaceous or hybrid materials. Furthermore, inert metal cores have very low $(<100 \Omega / \mathrm{m})$ axial resistance and are compatible with a wide range of further possible biofunctionalization strategies, to detect e.g. $\mathrm{pH}^{47}$, catecholamines ${ }^{48}$, or DNA ${ }^{49}$. Finally, through multi-step electrochemistry we created high rugosity surfaces in combination with a reversible redox couple at the electrode-tissue interface, resulting in an attractive and stable substrate for microstimulation purposes.

Low invasiveness allowed us to record single units on several adjacent jULIEs, despite the multiple penetrations (Fig. $\mathbf{2 g}$ ). In combination with the flexibility of geometric custom arrangement of recording sites, this could be used to tailor probe arrays for optimal unit isolation, maximizing unit yield ${ }^{50}$.

For understanding the brain, recording and stimulation in three dimensions at high speed, and at a large distributed scale is critical. Various imaging, electrical or genetic approaches are available, each challenged by particular limitations of scalability ${ }^{50}$. Currently, the technologies that not only might be closest to that goal but are also applicable to clinical challenges are electrical recording and stimulation techniques with invasiveness considered to be the central obstacle. Here, jULIE probes combine low invasiveness with high quality electrical recording and stimulation, thus offering a platform for interfacing with brain activity at brain-wide scale with single-cell resolution.

\section{Acknowledgements}

We thank Martyn Stopps for help with electronic design, Isabell Whiteley for technical help with histological processing, and Anatolii loisher for advice on glass-metal microwires. We also thank Lucy Collison and the EM STP team for help with SEM imaging, the Making Lab and the BRF for technical help. We also thank to Ecaterina Ware and Mahmoud Ardakani from Imperial College Faculty of Engineering, Department of Materials for their help with characterizing and imaging nanoAu and IrOx nanostructures. We thank Howard Marriage and Veronique Birault and the 
Translational team at the Crick for supporting development of jULIEs through the Idea 2 Innovation grant series. This work was also supported by the Francis Crick Institute which receives its core funding from Cancer Research UK (FC001153), the UK Medical Research Council (FC001153), an HFSP grant (RGP 00048/2013), an NIH BRAIN initiative grant (1U01NS094248-01) and the Wellcome Trust (FC001153) and the Medical Research Council (MC_UP_1202/5). Ede A. Rancz is a Sir Henry Dale Fellow (Wellcome, 104285/B/14/Z). Andreas Schaefer is a Wellcome Trust investigator (110174/Z).

\section{Dissemination}

jULIE probes in standard and custom layouts can be obtained from neurotrodics.com.

\section{Declaration of interest}

R Racz founded and holds shares in Nanotrodics, a company manufacturing composite ultramicroelectrodes for neurotechnological applications. AT Schaefer co-founded and holds shares in Paradromics, Inc, a company developing scalable electrophysiology.

\section{Materials and methods}

\section{Animal welfare}

All experiments were performed according to United Kingdom Home Office regulations (Animal, Scientific Procedures Act 1986) or the guidelines of the German animal welfare law and were approved by the local welfare committees and veterinarians.

\section{jULIE neural probes}

Glass-metal composite ultramicroelectrodes were fabricated by an adapted TaylorUlitovsky thermal drawing method ${ }^{8}$. Typically, a cylindrical borosilicate glass tube (OD $10 \mathrm{~mm}$, ID $6 \mathrm{~mm}$, Pyrex, Corning, UK) was loaded with a metal rod (Puratronic, Alfa Aesar, UK) and inductively heated (PowerCube 900 , CEIA, UK) to $850-1000{ }^{\circ} \mathrm{C}$ until a separating drop formed. The thermoformed part was removed and the flowing fiber was, at high speeds $(2 \mathrm{~km} / \mathrm{min})$, continuously spooled up on a drum as depicted in Figure 1a. This process resulted in electrically continuous conductive ultramicroelectrodes with outer diameters ranging from $10 \mu \mathrm{m}$ to $100 \mu \mathrm{m}$ with cores from $2 \mu \mathrm{m}$ to $10 \mu \mathrm{m}$ and lengths in the order of hundreds of meters.

Ultramicroelectrodes were bundled together using an Optima 1100 (Synthesis, India) winding machine and embedded in Crystalbond 509 (Agar, USA) a thermoplastic, dissolvable resin to fit custom 3D-printed polishing holders. jULIEs were sharpened at 30 degrees using a MetaServ 250 polisher equipped with a Vector head (Buehler, USA) and sequentially polished at 5 different levels using particles $(25 \mu \mathrm{m}, 9 \mu \mathrm{m}, 3 \mu \mathrm{m}, 1 \mu \mathrm{m}, 0.05 \mu \mathrm{m})$ suspended in water-based emulsion (Buehler, USA) for 40 seconds each. Sharpened wires were de-embedded by solubilization (1000:1 solid/liquid ratio) in Crystalbond 509 organic stripper (Agar, USA) for $24 \mathrm{~h}$ then washed with isopropanol and dried at $60^{\circ} \mathrm{C}$ overnight before long-term storage.

To record in vivo extracellular signals, we assembled jULIEs (Figure 1e-i, Supplementary Figure 1) into neural probe modules of 16,32 and 128 channels onto custom PCB (E44, LPKF Protomat, UK) using a modified instrument and bonding procedure (F\&S Bondtec, UK) which allowed in-situ read-out of connectivity and was equipped to remove glass insulation. Bonded and sharpened wires were then modified electrochemically as described below.

\section{NanoAu electrodeposition}

NanoAu was electrodeposited from a two-part aqueous cyanide bath containing $50 \mathrm{gL}^{-1}$ potassium dicyanoaureate $(\mathrm{I})\left(\mathrm{K}_{2}\left[\mathrm{Au}(\mathrm{CN})_{2}\right]\right)$ and $500 \mathrm{gL}^{-1} \mathrm{KH}_{2} \mathrm{PO}_{4}$ dissolved sequentially in ultrapure 
deionized water $(18 \mathrm{M} \Omega \cdot \mathrm{cm})(\mathrm{Tech}, \mathrm{UK})$ at $60^{\circ} \mathrm{C}$. All reagents were supplied by Sigma-Aldrich, UK and used without further purification. Prior to electrodeposition the polished substrate was washed with deionized water, rinsed with ethanol (90\%), wiped with a lint-free cloth (Kimwipes, Kimtech, UK) and dried at $50{ }^{\circ} \mathrm{C}$ for 1 hour in an oven (Memmert, Germany). The electrodeposition protocol was carried out with a multichannel potentiostat-galvanostat (VSP 300, Bio-Logic, France) controlled with EC-Lab software (Bio-Logic, France) in a three-electrode cell setup composed of the assembled gold jULIE probes as working electrodes $\left(\mathrm{W}_{\mathrm{E}}\right)$, a coiled 1 $\mathrm{mm}$ thick platinum wire (PT005150, 99.95\%, Goodfellow, US) as counter electrode $\left(C_{E}\right)$ and a $\mathrm{Ag} / \mathrm{AgCl}_{\mathrm{KCl}} \mathrm{KCM}_{3.5 \mathrm{M}}$ reference electrode (REF) supplied by BASi, USA (E vs. Normal Hydrogen Electrode $(\mathrm{NHE})=0.205 \mathrm{~V})$. The REF was kept separated from the bath by a glass tube containing the support electrolyte and a porous Vycor glass separator. For nanoAu deposition the $W_{E}$ potential was kept at $E_{\text {red }}=-1.1$ vs. REF for up to 180 seconds according to the desired hemisphere size as shown in Figure $1 \mathrm{~m}$. The electrodeposition bath was maintained at $60{ }^{\circ} \mathrm{C}$ using a thermostat under vigorous (500 rpm) stirring.

\section{IrOx electrodeposition}

The electrodeposition protocol was carried out from a modified electrolyte solution based on a formulation reported by (Kazusuke 1989; Meyer 2001) containing $10 \mathrm{gL}^{-1}$ iridium (IV) chloride hydrate (99.9\%, trace metal basis, Sigma-Aldrich, Germany), $25.3 \mathrm{gL}^{-1}$ oxalic acid dihydrate (reagent grade, Sigma-Aldrich, Germany), $13.32 \mathrm{gL}^{-1}$ potassium carbonate $(99.0 \%$, BioXtra, Sigma- Aldrich, Germany). Reagents were added sequentially to $50 \%$ of the solvent's volume firstly by dissolving $\mathrm{IrCl}_{4}$ in oxalic acid followed by the addition of $\mathrm{K}_{2} \mathrm{CO}_{3}$ over a 16-hour period until $\mathrm{pH}=12$ was reached. The electrolyte was aged approx. 20 days at room temperature in normal light conditions until the solution reached dark blue color. IrOx was electrodeposited using the instrumentation and setup described above (see section nanoAu Electrodeposition). The deposition protocol was composed of two consecutive stages combining cyclic voltammetry (CV) and a pulsed potentiostatic protocol (PP). Between protocols, the $\mathrm{W}_{\mathrm{E}}$ was kept at the open circuit voltage (OCV) for 180 seconds to allow equilibration. During $C V$ deposition the $W_{E}$ potential was cycled 50 times between $-0.5 \mathrm{~V}$ and $0.60 \mathrm{~V}$ vs. REF at $1 \mathrm{Vs}^{-1}$ in both anodic and cathodic directions. During the pulsed potentiostatic deposition the $\mathrm{W}_{\mathrm{E}}$ potential was stepped 250 times between $0 \mathrm{~V}$ to $0.60 \mathrm{~V}$ vs. REF in 1 second steps.

\section{jULIEs electrochemical characterization}

Electrochemical behavior of the sensor sites was monitored individually in the unmodified state (polished gold/glass surface), after modification with nanoAu, and after modification with nanoAu+IrOx. Multichannel jULIE modules were assembled and connected to the potentiostat using a custom PCB and matching connector plugs. Characterization was done in $150 \mathrm{mM}$ phosphate buffered saline using a 3-electrode cell-setup (see details for $C_{E}$ and REF above) for their cyclic voltammetry response and electrochemical impedance (EIS) profile. EIS measurements were performed by applying a $10 \mathrm{mV}$ sine-wave around the open circuit voltage $(\mathrm{OCV})$ in the frequency range $1 \mathrm{~Hz}$ to $100 \mathrm{kHz}$ with average 3 measurements per frequency and 5 repetitions for each channel. Using an identical cell setup, cyclic voltammetry response was recorded for individual channels by sweeping electrode potential from $-0.5 \mathrm{~V}$ and $0.6 \mathrm{~V}$ vs. REF with $100 \mathrm{mVs}^{-1}$ sweeping rate in both cathodic and anodic directions.

\section{FIB-SEM and STEM characterization}

Modified sensor sites were characterized for their microstructure, atomic lattices and chemical composition by field emission electron microscopy (FESEM) and scanning transmission electron microscopy (STEM) using a multipurpose 200 kV JEOL JEM-2100F TEM analytical electron 
microscope coupled with an Energy Dispersive X-ray Spectrometer (EDS) and Oxford Instruments INCA/Aztech EDS $80 \mathrm{~mm}$ X-Max detector system for elemental analysis with nanometer spatial resolution. A dual-beam FEI Helios $600 \mathrm{FIB} / \mathrm{SEM}$ system equipped with a gallium ion source operating in the accelerating voltage range $0.5-30 \mathrm{kV}$ and an Omniprobe ${ }^{\mathrm{TM}}$ micromanipulator was used to morphologically characterize and prepare samples for transmission electron microscopy (TEM) imaging. Sample preparation consisted of: (i) deposition of a protective platinum ( $\mathrm{Pt}$ ) layer by sputtering on to the specimens, (ii) milling a thin slice perpendicularly to the sample surface, (iii) extraction and gluing the specimen slice to a TEM grid, and (iv) further thinning of the sample with low-voltage focused ion-beams at grazing incidence until an electrontransparent region was obtained. These steps were repeated for both nanoAu and nanoAu+IrOx specimens as depicted in Supplementary figures 2 and 3.

\section{LFP modelling}

For simulation of the LFP around a detailed mitral cell model, we used a Neurolucida reconstruction of a mitral cell (IF04208 ${ }^{51}$ ). Ion channel densities for different domains (glomerular tuft, apical dendrite, lateral dendrite, soma, axon) were adapted from Rubin et al. 2006. To gain a more accurate picture of the field around the initial segment, a sodium channel density of $2000 \mathrm{pS} / \mu \mathrm{m}^{2}{ }^{52,53}$ was included in the first $5 \mu \mathrm{m}$ of the axon. The LFP was simulated at different locations with the line-source method ${ }^{54}$, with the LFPy Python package ${ }^{55}$.

\section{Histology}

Tissue integrity post jULIE insertion was determined by histological methods as described $^{34}$. In brief, after craniotomy, $0.2 \mathrm{ml}$ of $0.5 \%$ Evans Blue was injected into the tail vein. Prior to insertion, jULIEs were dipped into SP-DiO (Molecular Probes, OR, USA) and allowed to dry. After the jULIEs were removed from the brain the mouse was perfused with ice cold $4 \%$ PFA, the brain was harvested and stored in 4\% PFA overnight. Using a Vibratome (Leica, Germany), the brain was sliced into $100 \mu \mathrm{m}$ horizontal sections. Slices were stained with DAPI using a 1:1000 DAPI:PBS wash for 10 minutes, transferred to fresh PBS, mounted and sealed. Imaging was completed on a confocal microscope (Leica SP5).

\section{Extracellular recordings in the olfactory bulb}

To test jULIEs we performed recordings in vivo in the OB of mice. 4-6 weeks old mice were anaesthetized using a mixture of Ketamine/Xylazine (100 mg per $\mathrm{kg}$ of body weight) and xylazine (20 mg per $\mathrm{kg}$ for induction and $10 \mathrm{mg}$ per $\mathrm{kg}$ for maintenance) administered intraperitoneally and supplemented as required. Body temperature was maintained at $37^{\circ} \mathrm{C}$ using a feedback-regulated heating pad (FST, USA). The main olfactory bulb was accessed through a $2 \times 2 \mathrm{~mm}$ craniotomy window after fixation of the skull with a head-plate. The surface of the brain was protected by an imaging well containing Ringer's solution and caudally fixed $\mathrm{Ag} \mid \mathrm{AgCl}$ reference electrode. 
Using micromanipulators (Luigs \& Neumann, Germany) the jULIE probes were lowered to a depth of approximately $400 \mu \mathrm{m}$. Extracellular recordings were performed using a Tucker Davis RZ2 amplifier with a RA16AC-Z headstage or an Intan RHD2132 headstage on an OpenEphys amplifier (openephys.org).

Mice were presented with mixtures of odorants (Mixture 1: Ethyl butyrate \& 2-hexanone, Mixture 2: Eucalyptol \& Amayl accotate) (Sigma Aldrich, USA) diluted 1:5 with mineral oil. Recordings were taken from $400 \mu \mathrm{m}$ from the surface of the bulb. Units were isolated using either Spike2 (Cambridge Electronics Devices, Cambridge, UK) or Kilosort (Pachitariu 2016) and units with well-defined auto-correlograms were selected for further analysis. Units were found with distinct responses to different odors, that were stable across repeats.

\section{Extracellular recordings in the inferior colliculus}

Female C57BL/6JOlaHsd (Janvier, France) mice were anaesthetized with Avertin $(0.15$ $\mathrm{ml} / 10 \mathrm{~g})$. Additional doses $(0.03 \mathrm{ml} / 10 \mathrm{~g})$ were given as needed to maintain anesthesia. After anesthesia, the animal was fixed with blunt ear bars on a stereotaxic apparatus (Kopf, Germany). Body temperature was maintained at $36{ }^{\circ} \mathrm{C}$ with a feedback-regulated heating pad (ATC 1000, WPI, Germany). Vidisic eye gel (Bausch + Lomb GmbH, Germany) was used to prevent the eyes from drying out. A metal head-holder was glued to the skull $1.0 \mathrm{~mm}$ rostral to Lambda with methyl methacrylate resin (Unifast TRAD, GC). A craniotomy of $0.8 \mathrm{~mm} \times 1.0 \mathrm{~mm}$ with the center $0.85 \mathrm{~mm}$ from the midline and 0.75 caudal to Lambda was made to expose the left inferior colliculus. Dura was carefully removed and the surface of the brain protected with Saline (B. Braun, Germany). The inferior colliculus was identified by its position posterior to the transverse sinus and anterior to the sigmoid sinus. With a micromanipulator (Kopf, Germany), jULIEs were lowered vertically and advanced into the inferior colliculus.

The electrophysiological signal was amplified (HS-18-MM, Neuralynx, USA), sent to an acquisition board (Digital Lynx 4SX, Neuralynx, USA), and recorded with a Cheetah 32 Channel System (Neuralynx, USA). The voltage traces were acquired at a $32 \mathrm{kHz}$ sampling rate with a wide bandpass filter $(0.1 \pm 9,000 \mathrm{~Hz})$.

The sound was synthesized using MATLAB, produced by a USB interface (Octa capture, Roland, USA), amplified (Portable Ultrasonic Power Amplifier, Avisoft, Germany), and played with a freefield ultrasonic speaker (Ultrasonic Dynamic Speaker Vifa, Avisoft, Germany). The speaker was positioned $15 \mathrm{~cm}$ away from the right ear. Sound intensity was calibrated with a Bruël \& Kjaer microphone. For measuring the tonal receptive field, we used sound stimuli consisting of $30 \mathrm{~ms}$ pure tone pips with $5 \mathrm{~ms}$ rise/fall slope repeated at a rate of $2 \mathrm{~Hz}$. Thirty-two frequencies $(2 \mathrm{kHz}$ to $47 \mathrm{kHz}, 0.16$ octave spacing) were played in a pseudorandom order at intensities of 60 or 70 $\mathrm{dB}$. Each tone-intensity combination was played 5 times.

The recorded voltage signals were high-pass filtered at $500 \mathrm{~Hz}$. The root-mean-square (RMS) of the noise of each channel was calculated as the RMS level of the filtered trace during the first 10 seconds of recordings, which included spontaneous and evoked activity. To improve the signalto-noise ratio of the recording, the common average reference was calculated from all the functional channels and subtracted from each channel (Ludwig 2009). For multiunit analysis, spikes were detected as local minima below a threshold of 6 times the median absolute deviation of each channel. If the calculated value was higher than $-40 \mu \mathrm{V}$, the threshold was set to $-40 \mu \mathrm{V}$. To analyze the sound-driven responses, peri-stimulus time histograms (PSTHs) were built by aligning the signals at stimulus onset and calculating the number of spikes $/ \mathrm{ms}$. Isointensity tuning curves were built from the sum of spikes in an 80 ms window from stimulus onset, at each intensity as a function of frequency. 


\section{Extracellular recordings in the L5 of primary visual cortex}

Male C57BL/ 6 mice between 2-4 months old were used for acute recordings in the visual cortex. For surgery, mice were anaesthetized with isoflurane (3\% induction followed by $2 \%$ maintenance), and fixed on a stereotaxic apparatus (Model 940, David Kopf instruments, Germany) using ear bars. Body temperature was maintained at $36^{\circ} \mathrm{C}$ with a $\mathrm{DC}$ temperature regulation system (FHC, Inc. USA). A skin incision was made and the exposed skull was cleaned and dried. A metal headplate was cemented to the skull using dental cement (Super-Bond C\&B, Sun Medical, Japan). Animals were allowed to recover after surgery in their home cage. Analgesia ( $2 \mathrm{mg} / \mathrm{kg}$ Meloxicam with $0.1 \mathrm{mg} / \mathrm{kg}$ Buprenorphine) was provided. On the day of the recording, a craniotomy $1.5 \mathrm{~mm}$ long, 0.3-0.5 mm wide was made with small drill bits on the right hemisphere under isoflurane anesthesia (3\% induction followed by $2 \%$ maintenance). The long axis of the craniotomy was parallel with the lambda suture. The dura was removed and the craniotomy covered with a silicone-based sealant (Kwik-cast, World Precision Instruments). Following recovery from surgery (4-24 hours), the animal was lightly anaesthetized in 1.0-1.5\% isoflurane and head-fixed to the recording setup. The craniotomy was kept moist with cortex buffer ( $\mathrm{NaCl} 125 \mathrm{mM}, \mathrm{KCl} 5 \mathrm{mM}$, HEPES $10 \mathrm{mM}, \mathrm{MgSO}_{4} 2 \mathrm{mM}, \mathrm{CaCl}_{2} 2 \mathrm{mM}$, Glucose $10 \mathrm{mM}, \mathrm{pH}$ 7.4) and the jULIEs or Si-probe (A4X1-tet-3mm-150-121, Neuronexus, USA) was slowly ( 10-20 $\mu \mathrm{m} /$ minute) inserted into primary visual cortex using a micromanipulator (SM1, Luigs \& Neumann, Germany). Neural signals were recorded using a PZ2-32 preamplifier and an RZ2 BioAmp Processor (Tucker-Davis Technologies, USA). Data were acquired at $24.4 \mathrm{kHz}$ sampling rate and recorded using TDT's OpenEx software suite. The amplifier ground was connected to a screw implanted in the skull.

For visual stimulation full screen drifting gratings of 8 different directions (spatial frequency: $0.08 \mathrm{cpd}$; temporal frequency $2 \mathrm{~Hz}$; $2 \mathrm{~s}$ long, with $2 \mathrm{~s}$ grey screen inbetween), were presented in randomized order on a 27-inch LCD screen (E2711T, LG Electronics), placed $15-25 \mathrm{~cm}$ from the left eye. Gratings were generated and presented using MATLAB (Mathworks, USA) and the Psychophysics toolbox ${ }^{56}$.

Data were extracted and processed using custom written Matlab scripts. Neural signals were bandpass filtered at 300-5000 Hz for spike detection. Semi-automatic spike sorting was carried out using Klusta software with default parameters (Rossant 2016). Clusters with clear refractory period in the auto-correlogram (ISI violation $<0.5 \%$ ) and isolation distance $>20$ were classified as single units. Manual curation of resultant clusters was done using Phy ${ }^{26}$.

In vivo two-photon olfactory bulb wire insertion and imaging

Mice were anaesthetized using a mixture of Fentanyl/Midazolam/Medetomidine $(0.05$ $\mathrm{mg} / \mathrm{kg} / 5 \mathrm{mg} / \mathrm{kg} / 0.5 \mathrm{mg} / \mathrm{kg}$ ). The skull overlying the dorsal olfactory bulb was thinned using a dental drill and removed with forceps, the dura was peeled back using fine forceps. Body temperature was maintained at $37^{\circ} \mathrm{C}$ throughout the experiment using a feedback-controlled heating pad (FHC, Inc. USA). Sulforhodamine 101 (Sigma Aldrich, $100 \mu \mathrm{m}$ final concentration) was injected intraperitoneally to label blood vessels. Animals were then moved to a two-photon microscope (Scientifica Multiphoton VivoScope) coupled with a MaiTai Deep See laser (Spectra Physics, Santa Clara, CA) tuned to $940 \mathrm{~nm}(\sim 50 \mathrm{~mW}$ average power on the sample) for imaging. Images ( $512 \times 512$ pixels) were acquired with a resonant scanner at a frame rate of $30 \mathrm{~Hz}$ using a 16x 0.8 NA water-immersion objective (Nikon). For in vivo z-stack imaging, images were taken at a resolution of $512 \times 512$ pixels with $2 \mu \mathrm{m}$ z intervals. Wires were dip-coated in DiO (Sigma Aldrich) before insertion into the dorsal olfactory bulb using a micro manipulator (Scientifica, Uckfield, UK). Images were analyzed post hoc using ImageJ (NIH, Bethesda).

In vivo two-photon imaging of stimulation in the olfactory bulb 
Mice were prepared for imaging as described in the previous section. Wires were dipcoated in Dil (Sigma Aldrich) before insertion into the dorsal olfactory bulb using a micro manipulator (Scientifica, Uckfield, UK). A silver/silver chloride counter electrode was inserted into caudal parts of the imaging well. For electrical stimulation, typically $100 \mathrm{~ms}$ long step current pulses were applied repeatedly to the inserted microwire relative to the caudal reference electrode with a Digitimer NL800A stimulator while imaging at typically 6 z-planes ( $\partial z$ $=25 \mu \mathrm{m}$ ). Total frame rate was $30 \mathrm{~Hz}$, resulting in an effective volume repetition rate of $5 \mathrm{~Hz}$. Stimulation strength was varied between 5 and $85 \mu \mathrm{A}$ in $5 \mu \mathrm{A}$ steps and repeated 3-5 times for each stimulation strength. ROls were selected manually offline using custom written routines in ImageJ and data was exported for further analysis in Matlab.

\section{References}

1. Buzsáki, G. Large-scale recording of neuronal ensembles. Nat Neurosci 7, 446-451 (2004).

2. Doron, G. \& Brecht, M. What single-cell stimulation has told us about neural coding. Philos. Trans. R. Soc. Lond., B, Biol. Sci. 370, 20140204 (2015).

3. Jun, J. J. et al. Fully integrated silicon probes for high-density recording of neural activity. Nature 551, 232-236 (2017).

4. Salatino, J. W., Ludwig, K. A., Kozai, T. D. Y. \& Purcell, E. K. Glial responses to implanted electrodes in the brain. Nat Biomed Eng 1-16 (2017). doi:10.1038/s41551-017-0154-1

5. Seymour, J. P. \& Kipke, D. R. Neural probe design for reduced tissue encapsulation in CNS. Biomaterials 28, 3594-3607 (2007).

6. Du, Z. J. et al. Ultrasoft microwire neural electrodes improve chronic tissue integration. Acta Biomaterialia 53, 46-58 (2017).

7. Lo, M.-C. et al. Evaluating the in vivo glial response to miniaturized parylene cortical probes coated with an ultra-fast degrading polymer to aid insertion. J. Neural Eng. 15, 036002 (2018).

8. Taylor, G. F. Process and apparatus for making filaments. 1-6 (1928).

9. Baranov, S. A. A three-layer model of an amorphous microwire. Surf. Engin. Appl.Electrochem. 46, 271-275 (2010).

10. Edell, D. J., Toi, V. V., McNeil, V. M. \& Clark, L. D. Factors influencing the biocompatibility of insertable silicon microshafts in cerebral cortex. IEEE Trans. Biomed. Eng. 39, 635-643 (1992).

11. Obaid, A. et al. Massively Parallel Microwire Arrays Integrated with CMOS chips for Neural Recording. 9, 477-29 (2019).

12. Robinson, D. A. The Electrical Properties of Metal Microelectrodes. Proceedings of the IEEE 56, 1065-1071 (1968).

13. Loeb, G. E., Peck, R. A. \& Martyniuk, J. Toward the ultimate metal microelectrode. Journal of Neuroscience Methods 63, 175-183 (1995).

14. Ahuja, A. K., Behrend, M. R., Whalen, J. J., Humayun, M. S. \& Weiland, J. D. The Dependence of Spectral Impedance on Disc Microelectrode Radius. IEEE Trans. Biomed. Eng. 55, 1457-1460 (2008).

15. Kozai, T. D. Y. et al. Comprehensive chronic laminar single-unit, multi-unit, and local field potential recording performance with planar single shank electrode arrays. Journal of Neuroscience Methods 242, 15-40 (2015).

16. Fattahi, P., Yang, G., Kim, G. \& Abidian, M. R. A review of organic and inorganic biomaterials for neural interfaces. Adv. Mater. 26, 1846-1885 (2014). 
17. Yamanaka, K. Anodically electrodeposited iridium oxide films (AEIROF) from alkaline solutions for electrochromic display devices. Japanese Journal of Applied Physics 28, 632637 (1989).

18. Meyer, R. D., Cogan, S. F., Nguyen, T. H. \& Rauh, R. D. Electrodeposited iridium oxide for neural stimulation and recording electrodes. IEEE Trans Neural Syst Rehabil Eng 9, 2-11 (2001).

19. Steegstra, P., Busch, M., Panas, I. \& Ahlberg, E. Revisiting the Redox Properties of Hydrous Iridium Oxide Films in the Context of Oxygen Evolution. J. Phys. Chem. C 117, 2097520981 (2013).

20. Cogan, S. F. Neural stimulation and recording electrodes. Annual Review of Biomedical Engineering 10, 275-309 (2008).

21. Rácz, R., Manciulea, A. \& Ilea, P. Electrochemical behavior of metallic titanium in $\mathrm{MnO}_{2}$ electrosynthesis from spent battery leach liquors. Studia Universitatis Babes-Bolyai, Chemia 56, 211-222

22. Fiáth, R. et al. Slow insertion of silicon probes improves the quality of acute neuronal recordings. Sci. Rep. 9, 111 (2019).

23. Radu, M. \& Chernoff, J. An in vivo assay to test blood vessel permeability. $J$ Vis Exp e50062 (2013). doi:10.3791/50062

24. Gold, C., Henze, D. A., Koch, C. \& Buzsáki, G. On the origin of the extracellular action potential waveform: A modeling study. Journal of Neurophysiology 95, 3113-3128 (2006).

25. Bakkum, D. J. et al. The Axon Initial Segment is the Dominant Contributor to the Neuron's Extracellular Electrical Potential Landscape. Adv Biosyst 3, (2019).

26. Rossant, C. et al. Spike sorting for large, dense electrode arrays. Nat Neurosci 19, 634-641 (2016).

27. Pachitariu, M., Steinmetz, N., Kadir, S., Carandini, M. \& Kenneth D, H. Kilosort: realtime spike-sorting for extracellular electrophysiology with hundreds of channels. 222, 47-14 (2016).

28. Buzsáki, G. et al. Tools for Probing Local Circuits: High-Density Silicon Probes Combined with Optogenetics. Neuron 86, 92-105 (2015).

29. Kozai, T. D. Y. et al. Ultrasmall implantable composite microelectrodes with bioactive surfaces for chronic neural interfaces. Nature Materials 11, 1065-1073 (2012).

30. Saleem, A. B., Ayaz, A., Jeffery, K. J., Harris, K. D. \& Carandini, M. Integration of visual motion and locomotion in mouse visual cortex. Nature Publishing Group 16, 1864-1869 (2013).

31. Blanche, T. J., Spacek, M. A., Hetke, J. F. \& Swindale, N. V. Polytrodes: High-Density Silicon Electrode Arrays for Large-Scale Multiunit Recording. Journal of Neurophysiology 93, 2987-3000 (2005).

32. Niell, C. M. \& Stryker, M. P. Highly Selective Receptive Fields in Mouse Visual Cortex. Journal of Neuroscience 28, 7520-7536 (2008).

33. Guitchounts, G., Markowitz, J. E., Liberti, W. A. \& Gardner, T. J. A carbon-fiber electrode array for long-term neural recording. J. Neural Eng. 10, 046016 (2013).

34. Kollo, M. et al. CHIME: CMOS-hosted in-vivo microelectrodes for massively scalable neuronal recordings. bioRxiv 64, 1902-24 (2019).

35. Canales, A. et al. Multifunctional fibers for simultaneous optical, electrical and chemical interrogation of neural circuits in vivo. Nat Biotechnol 33, 277-284 (2015).

36. Luan, L. et al. Ultraflexible nanoelectronic probes form reliable, glial scar-free neural integration. Sci Adv 3, e1601966 (2017).

37. Ferro, M. D. et al. NeuroRoots, a bio-inspired, seamless Brain Machine Interface device for long-term recording. 2, 16093 
38. Musk, E.Neuralink. An integrated brain-machine interface platform with thousands of channels. 568, 493-12 (2019).

39. Guitchounts, G. \& Cox, D. 64-Channel Carbon Fiber Electrode Arrays for Chronic Electrophysiology. 113, 201517131

40. Hunt, D. L. et al. Multimodal in vivo brain electrophysiology with integrated glass microelectrodes. Nat Biomed Eng 5, 325 (2019).

41. Liu, J. et al. Syringe-injectable electronics. Nature Nanotechnology 10, 629-636 (2015).

42. Fu, T.-M., Hong, G., Viveros, R. D., Zhou, T. \& Lieber, C. M. Highly scalable multichannel mesh electronics for stable chronic brain electrophysiology. Proc. Natl. Acad. Sci. U.S.A. 114, E10046-E10055 (2017).

43. Ahmadi, N. et al. Towards a Distributed, Chronically-Implantable Neural Interface. in 719724 (IEEE). doi:10.1109/NER.2019.8716998

44. Deku, F. et al. Amorphous silicon carbide ultramicroelectrode arrays for neural stimulation and recording. J. Neural Eng. 15, 016007 (2018).

45. Angotzi, G. N. et al. SiNAPS: An implantable active pixel sensor CMOS-probe for simultaneous large-scale neural recordings. Biosensors and Bioelectronics 126, 355-364 (2019).

46. Ioisher, A. et al. Integration of Ge nanowire arrays in glass micro-fibers. Surf. Engin. Appl.Electrochem. 47, 103-106 (2011).

47. Steegstra, P. \& Ahlberg, E. Influence of oxidation state on the $\mathrm{pH}$ dependence of hydrous iridium oxide films. Electrochimica Acta 76, 26-33 (2012).

48. Ges, I. A., Currie, K. P. M. \& Baudenbacher, F. Electrochemical detection of catecholamine release using planar iridium oxide electrodes in nanoliter microfluidic cell culture volumes. Biosensors and Bioelectronics 34, 30-36 (2012).

49. Drummond, T. G., Hill, M. G. \& Barton, J. K. Electrochemical DNA sensors. Nat Biotechnol 21, 1192-1199 (2003).

50. Marblestone, A. H. Physical principles for scalable neural recording. 1-34 (2013). doi:10.3389/fncom.2013.00137/abstract

51. Fukunaga, I., Berning, M., Kollo, M., Schmaltz, A. \& Schaefer, A. T. Two distinct channels of olfactory bulb output. Neuron 75, 320-329 (2012).

52. Kole, M. H. P. \& Stuart, G. J. Signal processing in the axon initial segment. Neuron 73, 235247 (2012).

53. Lorincz, A. \& Nusser, Z. Cell-Type-Dependent Molecular Composition of the Axon Initial Segment. Journal of Neuroscience 28, 14329-14340 (2008).

54. Holt, G. R. \& Koch, C. Electrical interactions via the extracellular potential near cell bodies. $J$ Comput Neurosci 6, 169-184 (1999).

55. Lindén, H. et al. LFPy: A tool for biophysical simulation of extracellular potentials generated by detailed model neurons. Front. Neuroinform. 7, 41 (2014).

56. Brainard, D. H. The Psychophysics Toolbox. Spat Vis 10, 433-436 (1997). 